\title{
THE STRUCTURE OF EXPONENTIAL WEYL ALGEBRAS
}

\author{
P. L. ROBINSON
}

(Received 26 April 1991)

Communicated by P. Schultz

\begin{abstract}
We present structural properties of the complex associative algebra generated by the canonical commutation relations in exponential form. In particular, we show it to be a central simple algebra that lacks zero divisors and is not Noetherian on either side; in addition, we determine explicitly its units and its automorphisms.
\end{abstract}

1991 Mathematics subject classification (Amer. Math. Soc.): 16 D 30, 16 S 35, 16 U 60.

\section{Introduction}

The Weyl algebra with generators $p_{1}, \ldots, p_{m}, q_{1}, \ldots, q_{m}$ subject to the relations $\left[p_{i}, q_{j}\right]=\delta_{i j}$ and $\left[p_{i}, p_{j}\right]=\left[q_{i}, q_{j}\right]=0$ has been the recipient of much attention, not least because of its importance for the theory of enveloping algebras: see [1]. Its importance for quantum theory is probably exceeded by that of the "exponential" Weyl algebra, generated by the canonical commutation relations in exponential form.

Our aim here is to present some fundamental structural properties of the complex exponential Weyl algebra $A(V, \Omega)$ associated to a real symplectic vector space $(V, \Omega)$. We show that it is a central simple algebra without zero divisors and determine its group of units. We demonstrate that it is neither Noetherian nor Artinian: indeed, it has strictly ascending chains of principal left ideals and it has no minimal left ideals. We describe explicitly its full

(C) 1993 Australian Mathematical Society $0263-6115 / 93 \$ A 2.00+0.00$ 
automorphism group: this turns out to be a semidirect product of the complex character group of $V$ and the additive symplectic group of $(V, \Omega)$. Much of this structure theory follows from convexity properties of interest in their own right.

We remark that certain finitely-generated analogues of $A(V, \Omega)$ have been studied by a number of authors: see Jategaonkar [2] and McConnell and Pettit [5]. We remark also that the $C^{*}$-algebra completion of $A(V, \Omega)$ relative to a natural involution has been studied rather thoroughly: see Manuceau [3] and Slawny [8]; see also [4].

The work presented here developed from a University of Florida preprint [6] and was financially supported in part by the National Science Foundation.

\section{The structure of $A(V, \Omega)$}

Let $(V, \Omega)$ be a real symplectic vector space: the real vector space $V$ might be infinite dimensional; the skew bilinear form $\Omega$ is nonsingular in having trivial kernel. For $h=2 \pi \hbar$ a fixed positive real number, define $\varepsilon$ from $V \times V$ to the unit circle by

$$
x, y \in V \Rightarrow \varepsilon(x, y)=\exp \left\{\frac{1}{2 i \hbar} \Omega(x, y)\right\}
$$

and write $\omega$ for the square of $\varepsilon$.

The exponential Weyl algebra $A(V, \Omega)$ is the associative complex algebra of all finitely-supported maps $V \rightarrow \mathbb{C}$, the linear operations being defined pointwise and the product being given by

$$
(\phi \psi)(v)=\sum_{x+y=v} \varepsilon(x, y) \phi(x) \psi(y)
$$

for $\phi, \psi \in A(V, \Omega)$ and $v \in V$. Thus: $A(V, \Omega)$ is a twisted group algebra of the additive group underlying $V$, the twist being provided by the cocycle $\varepsilon$. For convenience, we shall henceforth drop the adjective "exponential" and refer to $A(V, \Omega)$ simply as the Weyl algebra of $(V, \Omega)$.

For $v \in V$ we denote by $\delta_{v}$ the element of $A(V, \Omega)$ taking value 1 at $v$ and 0 elsewhere. Note that

$$
x, y \in V \Rightarrow \delta_{x} \delta_{y}=\varepsilon(x, y) \delta_{x+y}
$$

so that in particular $\delta_{0}$ is the multiplicative identity of $A(V, \Omega)$ and $\delta_{v}$ is a unit with inverse $\delta_{-v}$ for each $v \in V$. Note also that $\left\{\delta_{v}: v \in V\right\}$ is of course a basis for $A(V, \Omega)$ as a vector space. 
We point out that if $\phi \in A(V, \Omega)$ and $u, v \in V$ then

$$
\left(\delta_{u} \phi \delta_{u}^{-1}\right)(v)=\omega(u, v) \phi(v) .
$$

Though elementary, this commutation rule enables us to establish rather directly that the algebra $A(V, \Omega)$ is central simple. Before embarking on the proof it is convenient to introduce further notation: namely, $\Sigma(\phi) \subset V$ to denote the (finite) support of $\phi \in A(V, \Omega)$. Note that if $u \in V$ then multiplication on either side by $\delta_{u}$ translates supports through $u$ so that $\Sigma\left(\delta_{u} \phi \delta_{u}^{-1}\right)=\Sigma(\phi)$ whenever $\phi \in A(V, \Omega)$.

We claim that the centre of $A(V, \Omega)$ consists precisely of all scalar multiples of $\delta_{0}$. To see this, let $\phi$ lie in the centre of $A(V, \Omega)$. If $v \in V$ is nonzero then there exists $u \in V$ with $\Omega(u, v) \notin h \mathbb{Z}$ so that $\omega(u, v) \neq 1$; now

$$
\omega(u, v) \phi(v)=\left(\delta_{u} \phi \delta_{u}^{-1}\right)(v)=\phi(v)
$$

forces $\phi(v)=0$. It follows that $\Sigma(\phi) \subset\{0\}$ so that $\phi$ is indeed a scalar multiple of $\delta_{0}$. The simplicity of $A(V, \Omega)$ is a consequence of the following result, itself of independent interest.

THEOREM. Let $J$ be an additive subgroup of $A(V, \Omega)$ invariant under the inner automorphism defined by $\delta_{u}$ for each $u \in V$. If $J$ is nonzero then $J$ contains a unit.

PROOF. Let $\phi$ be a nonzero element of $J$ for which the cardinality \# $\Sigma(\phi)$ is minimal. We claim that in fact $\# \Sigma(\phi)=1$, so that $\phi$ is a scalar multiple of some $\delta_{v}$ and hence a unit. For a contradiction, suppose $\Sigma(\phi)$ to contain distinct points $x$ and $y$. Choose $u \in V$ such that $\Omega(u, x) \in h \mathbb{Z}$ and $\Omega(u, y) \notin h \mathbb{Z}$. Observe that $J$ contains the element $\psi=\delta_{u} \phi \delta_{u}^{-1}-\phi$ and that $\Sigma(\psi) \subset \Sigma(\phi)$. On the one hand,

$$
\phi(x)=(\omega(u, x)-1) \phi(x)=0
$$

so that $\Sigma(\psi) \neq \Sigma(\phi)$; on the other hand,

$$
\psi(y)=(\omega(u, y)-1) \phi(y) \neq 0
$$

so that $\psi \neq 0$. Thus: the nonzero $\psi \in J$ has $\# \Sigma(\psi)<\# \Sigma(\phi)$ and so contradicts our choice of $\phi$.

As promised, we now have the central simplicity of the Weyl algebra. 
THEOREM. The Weyl algebra $A(V, \Omega)$ is central simple.

PROOF. We know already that the centre of $A(V, \Omega)$ comprises all scalar multiples of the identity. Any ideal of $A(V, \Omega)$ is certainly an additive subgroup invariant under all inner automorphisms; if it contains a unit then it is all of $A(V, \Omega)$.

This result has the usual consequences: for example, a nonzero homomorphism from $A(V, \Omega)$ is automatically injective: in particular, a nonzero representation of $A(V, \Omega)$ is automatically faithful.

Further structural properties of the Weyl algebra follow from a convexity analysis of the supports of its elements. The foundation of this analysis is the following.

THEOREM. If $\phi, \psi \in A(V, \Omega)$ are nonzero then

$$
\Sigma(\phi \psi) \subset \Sigma(\phi)+\Sigma(\psi)
$$

and both sides have the same convex hull in $V$.

PROOF. The stated inclusion is plain from the definition of multiplication in $A(V, \Omega)$. In order to complete the proof, it suffices to establish that every extreme point of $\Sigma(\phi)+\Sigma(\psi)$ lies in $\Sigma(\phi \psi)$; for this, it is enough to show that each such extreme point is uniquely a sum of elements from $\Sigma(\phi)$ and $\Sigma(\psi)$. To this end, let $x, x^{\prime} \in \Sigma(\phi)$ and $y, y^{\prime} \in \Sigma(\psi)$ with $x+y=x^{\prime}+y^{\prime}$ an extreme point of $\Sigma(\phi)+\Sigma(\psi)$; then

$$
x+y=\frac{1}{2}\left(x+y^{\prime}\right)+\frac{1}{2}\left(x^{\prime}+y\right)
$$

is a convex combination of two elements in $\Sigma(\phi)+\Sigma(\psi)$ which are distinct unless $x=x^{\prime}$ and $y=y^{\prime}$.

Denote by $e(\phi)$ the number of extreme points in the (finite) support $\Sigma(\phi)$ of $\phi \in A(V, \Omega)$. Of course, $e(\phi)=0$ iff $\phi=0$ and $e(\phi)=1$ iff $\phi$ is a nonzero scalar multiple of $\delta_{v}$ for some $v \in V$. In terms of this notation, the preceding theorem has the following numerical consequence for support convexity $e$.

THEOREM. If $\phi, \psi \in A(V, \Omega)$ are nonzero then $e(\phi \psi) \geq e(\phi) \vee e(\psi)$. 
PROOF. We must show that $e(\phi \psi) \geq e(\phi)$ and $e(\phi \psi) \geq e(\psi)$; by symmetry it is enough to show that $e(\phi \psi) \geq e(\phi)$. Let $x$ be an extreme point of $\Sigma(\phi)$ and choose a linear functional $f \in V^{*}$ such that $f(x)>f(\xi)$ for all $\xi \in \Sigma(\phi)$ except $\xi=x$. Among all points in $\Sigma(\psi)$ at which $f$ is maximized, select $y$ to be an extreme point of $\Sigma(\psi)$. We claim that $x+y$ is an extreme point of $\Sigma(\phi)+\Sigma(\psi)$. To see this, let $x^{\prime}, x^{\prime \prime} \in \Sigma(\phi)$, let $y^{\prime}, y^{\prime \prime} \in \Sigma(\psi)$, let $\lambda^{\prime}, \lambda^{\prime \prime}$ be positive real numbers with sum unity and assume

$$
x+y=\lambda^{\prime}\left(x^{\prime}+y^{\prime}\right)+\lambda^{\prime \prime}\left(x^{\prime \prime}+y^{\prime \prime}\right) .
$$

In the resulting equation

$$
f(x)-\lambda^{\prime} f\left(x^{\prime}\right)-\lambda^{\prime \prime} f\left(x^{\prime \prime}\right)=\lambda^{\prime} f\left(y^{\prime}\right)+\lambda^{\prime \prime} f\left(y^{\prime \prime}\right)-f(y)
$$

the left side is nonnegative whilst the right side is nonpositive, whence both sides vanish. From our choice of $f$ we see that $x^{\prime}=x^{\prime \prime}=x$ so that $y=\lambda^{\prime} y^{\prime}+\lambda^{\prime \prime} y^{\prime \prime}$ and therefore $y^{\prime}=y^{\prime \prime}=y$ by extremity. Thus: to each extreme point $x \in \Sigma(\phi)$ there corresponds an extreme point $x+y$ of $\Sigma(\phi)+\Sigma(\psi)$ with $y \in \Sigma(\psi)$. This correspondence is necessarily injective: if $x_{0} \in \Sigma(\phi)$ and $y_{0} \in \Sigma(\psi)$ with $x+y=x_{0}+y_{0}$ then

$$
f(x)-f\left(x_{0}\right)=f\left(y_{0}\right)-f(y)
$$

as before both sides vanish, so that $x=x_{0}$ and $y=y_{0}$. Our proof is now complete.

As the first algebraic inference from our analysis of support convexity, the Weyl algebra lacks zero divisors and so of course lacks nontrivial idempotents. Indeed, if $\phi, \psi \in A(V, \Omega)$ are nonzero then from $e(\phi \psi) \geq e(\phi) \vee e(\psi)$ it follows at once that $\phi \psi$ is nonzero.

THEOREM. $A(V, \Omega)$ has no zero divisors.

Our analysis of support convexity also allows us to identify the units of the Weyl algebra. In fact, let $\phi$ and $\psi$ in $A(V, \Omega)$ satisfy $\phi \psi=\delta_{0}$. From

$$
1=e\left(\delta_{0}\right)=e(\phi \psi) \geq e(\phi) \vee e(\psi)
$$

we infer that $e(\phi)=e(\psi)=1$ and therefore that $\phi$ and $\psi$ are scalar multiples of elements in $\left\{\delta_{v}: v \in V\right\}$. 
THEOREM. The units in $A(V, \Omega)$ are precisely the elements $\left\{\lambda \delta_{v}: 0 \neq \lambda \in \mathbb{C}\right.$, $v \in V\}$ having point support.

We are now in a position to address the issue of whether or not the Weyl algebra is Noetherian. For $0 \neq u \in V$ and for $n \in \mathbb{Z}$ let $J_{n}$ denote the principal left ideal of $A(V, \Omega)$ generated by $\phi_{n}=\delta_{0}-\delta_{u / 2^{n}}$ and write $\psi_{n}=\delta_{0}+\delta_{u / 2^{n}}$. In view of the factorization $\psi_{n} \phi_{n}=\phi_{n-1}$ it is clear that $\left(J_{n}\right)_{n=1}^{\infty}$ is increasing. Further, $\phi_{n} \notin J_{n-1}$ since $A(V, \Omega)$ lacks zero divisors and $\psi_{n}$ is not a unit; thus $\left(J_{n}\right)_{n=1}^{\infty}$ increases strictly. Consequently, the Weyl algebra is neither left Noetherian nor (by symmetry) right Noetherian.

THEOREM. $A(V, \Omega)$ is not (left or right) Noetherian: indeed, it contains strictly ascending chains of principal (left, right) ideals.

Failing to be Noetherian, the Weyl algebra a fortiori fails to be Artinian. More is true: the Weyl algebra lacks minimal one-sided ideals. To see this, let $J$ be a nonzero left ideal in $A(V, \Omega)$ and write $e(J)$ to denote the minimum $\wedge\{e(\psi): 0 \neq \psi \in J\}$. If we choose $\phi \in J$ to satisfy $e(\phi)>e(J)$ then the left ideal $J \cdot \phi$ satisfies

$$
e(J \cdot \phi) \geq e(\phi)>e(J)
$$

and is therefore properly included in $J$.

THEOREM. $A(V, \Omega)$ is not Artinian: indeed, it has no minimal one-sided ideals.

Knowing all the units of the Weyl algebra, we can actually determine its full automorphism group. To this end, let $T$ be an algebra automorphism of $A(V, \Omega)$. Since $T$ must map units to units, it determines maps $F V \rightarrow V$ and $f V \rightarrow \mathbb{C}^{*}$ such that

$$
v \in V \Rightarrow T\left(\delta_{v}\right)=f(v) \delta_{F(v)} ;
$$

of course, since $T$ is bijective so is $F$. If $x, y \in V$ then

$$
\begin{aligned}
T\left(\delta_{x}\right) T\left(\delta_{y}\right) & =f(x) f(y) \varepsilon(F x, F y) \delta_{F x+F y} \\
T\left(\delta_{x} \delta_{y}\right) & =\varepsilon(x, y) f(x+y) \delta_{F(x+y)} .
\end{aligned}
$$

Consequently, if $x, y \in V$ then we have the following two relations:

$$
\begin{aligned}
F(x+y) & =F(x)+F(y) \\
\varepsilon(x, y) f(x+y) & =f(x) f(y) \varepsilon(F x, F y) .
\end{aligned}
$$


The first of these relations tells us that $F$ is an automorphism of the additive group $V$; equivalently, $F$ is an automorphism of $V$ as a rational vector space. As a result, $F$ acts on the cohomology groups of $V$; in particular, those with coefficients in the trivial $V$-module $\mathbb{C}^{*}$. The second of our relations tells us that the cohomology class $[\varepsilon] \in H^{2}\left(V ; \mathbb{C}^{*}\right)$ is fixed under $F$. In fact, we can say more: $F$ actually fixes $\Omega$; we see this as follows.

Interchanging $x, y \in V$ in the equation

$$
\frac{f(x+y)}{f(x) f(y)}=\frac{\varepsilon(F x, F y)}{\varepsilon(x, y)}
$$

fixes the left side and inverts the right. It follows that if $x, y \in V$ then $\varepsilon(F x, F y) / \varepsilon(x, y)$ has value \pm 1 ; equivalently,

$$
x, y \in V \Rightarrow \Omega(F x, F y)-\Omega(x, y) \in h \mathbb{Z} .
$$

If $\Omega(F x, F y) \neq \Omega(x, y)$ for some $x, y \in V$ then the $\mathbb{Q}$-linearity of $F$ forces $\Omega(F(q x), F y)-\Omega(q x, y) \notin h \mathbb{Z}$ for sufficiently small nonzero $q \in \mathbb{Q}$. Thus: if $x, y \in V$ then $\Omega(F x, F y)=\Omega(x, y)$ and so $f(x+y)=f(x) f(y)$. We record our findings in the following form.

THEOREM. The algebra automorphisms of $A(V, \Omega)$ are precisely those selfmaps $T$ given by

$$
v \in V \Rightarrow T\left(\delta_{v}\right)=f(v) \delta_{F(v)}
$$

where $f$ is a complex character of the additive group $V$ and $F$ is an additive automorphism of $V$ fixing $\Omega$.

Thus: the automorphism group of $A(V, \Omega)$ is the semidirect product of the group $\operatorname{Sp}_{+}(V, \Omega)$ of additive automorphisms of $(V, \Omega)$ by its natural action on the complex character group $\mathrm{Ch}(V)$ of $V$ : there is a canonically split short exact sequence

$$
1 \rightarrow \mathrm{Ch}(V) \rightarrow \operatorname{Aut} A(V, \Omega) \rightarrow \mathrm{Sp}_{+}(V, \Omega) \rightarrow 1 .
$$

Of course, the inner automorphisms of $A(V, \Omega)$ constitute a subgroup of $\mathrm{Ch}(V)$ isomorphic to $V$ and comprising those $T$ for which $F=I$ and $f=\omega(u, \cdot)$ for some $u \in V$.

In fact, the arguments leading to our determination of the automorphisms of the Weyl algebra can be pressed a littler further. Let $\left(V^{\prime}, \Omega^{\prime}\right)$ be another real symplectic vector space and let $T$ be an algebra isomorphism from $A(V, \Omega)$ 
to $A\left(V^{\prime}, \Omega^{\prime}\right)$. Arguing as for the theorem, there exist a complex character $f V \rightarrow \mathbb{C}^{\bullet}$ and an additive isomorphism $F(V, \Omega) \rightarrow\left(V^{\prime}, \Omega^{\prime}\right)$ such that $T\left(\delta_{v}\right)=f(v) \delta_{F(v)}$ for all $v \in V$. Now, if $V$ is finite dimensional and $S$ is a symplectic basis for $(V, \Omega)$ then $F(S)$ is a symplectic basis for $\left(V^{\prime}, \Omega^{\prime}\right)$ so that $\operatorname{dim} V^{\prime}=\operatorname{dim} V$. Thus the (finite) dimension of $V$ is an algebraic invariant of $A(V, \Omega)$; see [7] for an alternative proof of this fact based on a study of maximal abelian subalgebras of $A(V, \Omega)$.

It is of some interest consider invariance properties of numerical quantities attached to elements of the Weyl algebra. Support cardinality is of course invariant under each automorphism of the Weyl algebra: if $T \in \operatorname{Aut} A(V, \Omega)$ determines $F \in \operatorname{Sp}_{+}(V, \Omega)$ then $\Sigma(T(\phi))=F(\Sigma(\phi))$ and therefore $\# \Sigma(T \phi)=\# \Sigma(\phi)$ for all $\phi \in A(V, \Omega)$. Support convexity is invariant under those automorphisms $T$ of $A(V, \Omega)$ for which the corresponding symplectic automorphism $F$ is $\mathbb{R}$-linear: if $\phi \in A(V, \Omega)$ then $e(T \phi)=e(\phi)$ for such $T$.

Recall that support convexity $e$ is rather well-behaved relative to multiplication; in contrast, support cardinality $\# \Sigma$ is quite badly behaved. It is natural to ask whether we can attach to elements of the Weyl algebra a numerical quantity combining the good behaviour of support cardinality under automorphisms and the good behaviour of support convexity under multiplication. In fact, such a numerical quantity can be defined as follows.

For $\phi \in A(V, \Omega)$ we denote by $e_{+}(\phi)$ the number of rationally extreme points in its (finite) support $\Sigma(\phi)$. Of course, $e_{+}$dominates $e$; furthermore, the value of $e_{+}$can be arbitrarily large for a fixed value of $e$ greater than one. Since additive automorphisms of $V$ are rationally linear, $e_{+}$is certainly invariant under the full automorphism group of $A(V, \Omega)$. Though it is not quite obvious, $e_{+}$is well behaved relative to multiplication; a proof of this fact runs as follows.

Regard $V$ as a rational vector space and extend to real scalars; denote the resulting realification by $V^{\mathbb{R}}$.

We claim that any chosen rationally extreme point of $\Sigma(\phi) \subset V$ is also a real extreme point of $\Sigma(\phi) \subset V^{\mathbb{R}}$. In order to verify this, we may (rationally) translate so as to arrange that the chosen point is 0 . It is enough to show that if 0 is a real convex combination from $\Sigma(\phi)-\{0\}$ then 0 is actually a rational convex combination from $\Sigma(\phi)-\{0\}$. Thus: let $0=\lambda_{0} x_{0}+\cdots+\lambda_{n} x_{n}$ be a shortest possible expression for 0 as a real convex combination of nonzero vectors $x_{0}, \ldots, x_{n}$ in $\Sigma(\phi) \subset V^{\mathbb{R}}$ with positive real coefficients $\lambda_{0}, \ldots, \lambda_{n}$ having sum unity. Let $\left\{x_{0}, \ldots, x_{n}\right\}$ have rational span $X \subset V$ and real span $X^{\mathbb{R}} \subset V^{\mathbb{R}}$; note that the rational dimension of $X$ and the real dimension of $X^{\mathbb{R}}$ agree. In fact, this common dimension is $n$ : otherwise, the Caratheodory 
lemma would yield a shorter expression for 0 as a real convex combination from $\Sigma(\phi)-\{0\}$.

Identify $X \subset X^{\mathbb{R}}$ with $\mathbb{Q}^{n} \subset \mathbb{R}^{n}$ for notational convenience. The rational matrix $\mathbb{X}$ with columns $x_{0}, \ldots, x_{n}$ has rank $n$ and so has nullity 1 , over either $\mathbb{Q}$ or $\mathbb{R}$. The equations $\mathfrak{X} \cdot \lambda=0$ and $\lambda_{0}+\cdots+\lambda_{n}=1$ now force $\lambda_{0}, \ldots, \lambda_{n}$ to be rational.

Our claim is vindicated: rationally extreme points of $\Sigma(\phi) \subset V$ are really extreme points of $\Sigma(\phi) \subset V^{\mathbb{R}}$. The validity of our claim justifies passing from rational scalars to real scalars in the argument that follows.

Let $\phi, \psi \in A(V, \Omega)$ be nonzero. Let $x$ be a rationally extreme point of $\Sigma(\phi) \subset V$; from above, $x$ is then a real extreme point of $\Sigma(\phi) \subset V^{\mathbb{R}}$. Choose a real-linear functional $f$ on $V^{\mathbb{R}}$ with $f(x)>f(\xi)$ for all $\xi \in \Sigma(\phi)$ except $\xi=x$ and choose a real extreme point $y$ of $\Sigma(\phi) \subset V^{\mathbb{R}}$ at which $f$ is maximized. The point $x+y$ is then real extreme for $\Sigma(\phi)+\Sigma(\psi) \subset V^{\mathbb{R}}$ and therefore rationally extreme for $\Sigma(\phi)+\Sigma(\psi) \subset V$; further, the assignment of $x+y$ to $x$ is necessarily injective. In this way, we arrive at the following promised result.

THEOREM. If $\phi, \psi \in A(V, \Omega)$ are nonzero then $e_{+}(\phi \psi) \geq e_{+}(\phi) \vee e_{+}(\psi)$.

\section{References}

[1] J. Dixmier, Enveloping algebras (North-Holland, Amsterdam, 1977).

[2] V. A. Jategaonkar, 'A multiplicative analog of the Weyl algebra', Comm. Algebra 12 (1984), $1669-1688$.

[3] J. Manuceau, ' $C^{*}$-algèbre des relations de commutation', Ann. Inst.H.Poincaré (A) 8 (1968), 139-161.

[4] J. Manuceau, M. Sirugue, D. Testard and A. Verbeure, 'The smallest $C^{*}$-algebra for canonical commutation relations', Comm. Math. Phys. 32 (1973), 231-243.

[5] J. C. McConnell and J. J. Pettit, 'Crossed products and multiplicative analogues of Weyl algebras', J. London Math. Soc. 38 (1988), 47-55.

[6] P. L. Robinson, 'The exponential Weyl algebra', University of Florida, preprint, 1988.

[7] _ ' 'Isomorphic exponential Weyl algebras', Glasgow Math. J. 33 (1991), 7-10.

[8] J. Slawny, 'On factor representations and the $C^{*}$-algebra of canonical commutation relations', Comm. Math. Phys. 24 (1972), 151-170.

Department of Mathematics

University of Florida

Gainesville, FL 32611

USA 